\title{
Otimização Mono-objetivo no Controle do Mosquito Aedes aegypti por meio de um Modelo de Duas Populações com Influência da Precipitação
}

\author{
L.S.B. SILVA ${ }^{1}$, A.S.V. VASCONCELOS ${ }^{1}$, A.L. SANCHES ${ }^{2}$, R.T.N. CARDOSO ${ }^{3}$, \\ J.L.A. FERNANDES ${ }^{3}$ e A.E. EIRAS ${ }^{4}$
}

Recebido em 19 de dezembro de 2017 / Aceito em 16 de dezembro de 2018

\begin{abstract}
RESUMO. Arboviroses transmitidas pelo Aedes aegypti como a dengue, a chikungunya, a zika e a febre amarela têm afetado uma parte crescente da população mundial ao longo dos anos. Uma vez que não existem vacinas para todos os arbovírus transmitidos por este vetor, o controle da população do vetor é a abordagem considerada viável para reduzir a disseminação das arboviroses. O presente trabalho tem como objetivo estudar e comparar a eficácia entre a realização de três aplicações de controle mecânico na população imatura (ovos, larvas e pupas) e a realização de três aplicações de controle químico na população de fêmeas em fase de oviposição do A. aegypti. O controle é aplicado durante 7 dias em diferentes épocas do ano: Primavera-Verão, Verão e Verão-Outono. O modelo entomológico que descreve a dinâmica das fases do A. aegypti possui parâmetros dependentes da precipitação. A população de fêmeas em fase de oviposição do modelo foi confrontada com dados amostrais de Índice Médio de Fêmeas Aedes coletados por meio de 245 armadilhas MosquiTRAP ${ }^{\circledR}$ espalhadas na área urbana da cidade de Lavras. O Algoritmo Genético Real Polarizado foi utilizado a fim de otimizar a intensidade e o tempo inicial de aplicação do controle degrau em relação aos custos de controle e aos custos relacionados à infestação do mosquito. Durante a aplicação das três formas de intervenções de controle, o número de mosquitos diminuiu ao longo do tempo e a intervenção Verão-Outono apresentou a melhor eficiência e menor custo. Adicionalmente, o número de descendentes basal do mosquito sofreu um impacto negativo dos parâmetros relacionados à mortalidade e um impacto oposto dos demais parâmetros entomológicos do mosquito.
\end{abstract}

Palavras-chave: controle, Aedes aegypti, Algoritmo Genético Real Polarizado.

\footnotetext{
*Autor correspondente: Lillia dos Santos Barsante Silva - E-mail: lilliabarsante@gmail.com - http://orcid.org/ 0000-0002-5615-7870

${ }^{1}$ Programa de Pós-graduação de Modelagem Matemática e Computacional, CEFET-MG, Centro Federal de Educação Tecnológica de Minas Gerais, Av. Amazonas, 5253, 30421-169, Belo Horizonte, MG, Brasil. E-mail: lilliabarsante@gmail.com, amalia.soares17@gmail.com

${ }^{2}$ Departamento de Engenharia, CEFET-MG, Centro Federal de Educação Tecnológica de Minas Gerais, Av. Amazonas, 5253, 30421-169, Belo Horizonte, MG, Brasil. E-mail: analuizatrz@gmail.com, rodrigoc@ des.cefetmg.br

${ }^{3}$ Departamento de Física e Matemática, CEFET-MG, Centro Federal de Educação Tecnológica de Minas Gerais, Av. Amazonas, 5253, 30421-169, Belo Horizonte, MG, Brasil. E-mail: acebal@dppg.cefetmg.br

${ }^{4}$ Departamento de Parasitologia, UFMG, Universidade Federal de Minas Gerais, Av. Antônio Carlos, 6627, 31270-901, Belo Horizonte, MG, Brasil.
} 


\section{INTRODUÇÃO}

Tornou-se mundialmente crescente o número de pessoas infectadas por dengue, febre amarela, febre chikungunya e zika vírus [20]. Estas doenças apresentam relevante impacto em saúde pública em diversas localidades e apenas a febre amarela possui uma vacina preventiva de fácil acesso à população. A transmissão destas arboviroses ao homem ocorre por meio da picada das fêmeas infectadas dos mosquitos do gênero Aedes, entre os quais a espécie Aedes aegypti figura como seu principal vetor [5]. O ciclo de vida do mosquito é composto por duas fases: imatura (ovos, larvas e pupas) e alada (mosquitos adultos machos e fêmeas). Em condições ambientais favoráveis, os ovos desenvolvem-se em larvas, que, por sua vez se transformam em pupas e, em seguida, em mosquitos adultos. O aumento dos índices pluviométricos, temperatura e umidade são fatores que favorecem a infestação pelos mosquitos. Consequentemente, no Brasil, a incidência das arboviroses transmitidas pelo $A$. aegypti ${ }^{1}$ tem aumento no Verão e Outono. Portanto, o número de casos das arboviroses apresentam um padrão sazonal [7]. Uma vez que não existem vacinas para todos os arbovírus transmitidos por este vetor, o controle da população do vetor, ou controle vetorial, é a abordagem recomendada para reduzir a disseminação das arboviroses. Entretanto, em muitos municípios brasileiros estes programas apresentam baixa eficácia devido a problemas de gerenciamento, interrupções e limitações técnico-científicas [22]. Devido à escassez de recursos, a maioria dos gestores concentram campanhas educativas e de ações de controle do mosquito no Verão. Contudo, há que se perguntar se esta estação é o momento em que o controle é mais eficaz, ou se outras estações devem ser consideradas para distribuir as ações de controle ao longo do ano.

Modelos entomológicos associam a infestação do A. aegypti a variáveis meteorológicas. A dependência com a precipitação trata-se de um assunto ainda pouco estudado na literatura, uma vez que o comportamento das chuvas é complexo, o que torna a realização de experimentos muito complexa. Contudo, alguns trabalhos sugerem por meio de simulações numéricas que a precipitação mostra-se um preditor positivo e significativo para a abundância de fêmeas em fase de oviposição do mosquito $[1,6,3]$. De forma semelhante, modelos de otimização juntamente com modelos entomológicos têm sido desenvolvidos para avaliar a eficácia de intervenções de controle no ciclo de vida do mosquito. Diversas heurísticas têm sido utilizadas na resolução destes problemas. Tais como, o Firefly Algorithm (FA) foi desenvolvido por [33] e utilizado por [21] para minimizar a população de $A$. aegypti em um dado intervalo de tempo por meio de estratégias ótimas de controle químico (inserção de inseticidas na população de machos naturais e fêmeas do mosquito) e controle biológico (inserção de machos estéreis no ambiente com infestação de fêmeas dos mosquito). O Algoritmo Genético Real Polarizado (AGRP) foi desenvolvido por [24] e utilizado por [27] para minimizar a população do A. aegypti em um dado intervalo de tempo por meio de estratégias ótimas de controle químico (inserção de inseticidas) nas fases imatura e alada de fêmeas do mosquito. O Variable Neighborhood Search (VNS) foi desenvolvido por [18] e utilizado por [13] para minimizar a população do A. aegypti em um dado intervalo de tempo por meio de estratégias ótimas de controle na fase imatura do mosquito.

\footnotetext{
${ }^{1}$ Ao longo do texto foi utilizado A. aegypti como abreviação da palavra Aedes aegypti.
} 
Neste trabalho foi realizado o estudo de um problema de otimização para avaliar a eficácia de se realizar três aplicações de controle mecânico na população imatura (ovos, larvas e pupas) e três aplicações de controle químico na população de fêmeas em fase de oviposição do A. aegypti, durante o tempo de 7 dias nas intervenções Primavera-Verão, Verão e Verão-Outono na cidade de Lavras (Minas Gerais, Brasil). Neste contexto, buscou-se otimizar com o uso do AGRP a intensidade e o tempo inicial de aplicação do respectivo controle em relação aos custos de controle e aos custos relacionados à infestação do mosquito. Por meio da dependência do modelo entomológico, o problema de otimização é estabelecido de modo a considerar a influência da precipitação no desenvolvimento do mosquito. O AGRP foi utilizado para resolver o problema de otimização.

\section{MODELO ENTOMOLÓGICO}

O modelo representa duas populações do A. aegypti, a população imatura (ovos, larvas e pupas) - $I(t)$ e a população de fêmeas em fase de oviposição - $F(t)$, de modo que parte dos parâmetros entomológicos do mosquito sejam dependentes da precipitação acumulada em cada unidade de tempo $p(t)$ de uma determinada área geográfica. Optou-se pela redução do número de populações do modelo a fim de se controlar a complexidade do algoritmo. Contudo, houve o cuidado de tentar recuperar o realismo do comportamento de trabalhos anteriores $[1,3]$.

As populações de mosquitos foram consideradas como fração da capacidade do meio C. A população de fêmeas em fase de oviposição $F(t)$ contribui depositando seus ovos viáveis a uma taxa de oviposição por unidade de indivíduo dada por $f \phi(p)\left(1-\frac{I(t)}{C}\right)$. A expressão dentro dos parênteses constitui um fator do tipo logístico que promove uma redução da taxa de oviposição por unidade de fêmea $\phi(p)$ em criadouros com alta densidade de mosquitos na fase imatura [19]. A taxa de desenvolvimento da população imatura para a população de fêmeas em fase de oviposição é dada por $\gamma \alpha(p)$. As taxas de mortalidade natural e adicional por unidade de indivíduo específica de cada compartimento são dadas por $\mu(p)$ e $u$, respectivamente. O valor da mortalidade adicional $u_{I}$ corresponde ao percentual da população imatura (ovos, larvas e pupas) do mosquito a ser atingida pela ação de controle mecânico, durante determinado instante de tempo. $\mathrm{O}$ valor da mortalidade adicional $u_{F}$ corresponde ao percentual da população de fêmeas em fase de oviposição do mosquito a ser atingida pela ação de controle químico, durante determinado instante de tempo.

O sistema de equações diferenciais não lineares que representa a dinâmica das populações do $A$. aegypti com dependência da precipitação é descrito por:

$$
\left\{\begin{aligned}
\frac{d I}{d t} & =f \phi(p)\left(1-\frac{I}{C}\right) F-\left(\alpha(p)+\mu_{I}(p)+u_{I}\right) I \\
\frac{d F}{d t} & =\gamma \alpha(p) I-\left(\mu_{F}(p)+u_{F}\right) F
\end{aligned}\right.
$$

com condições iniciais $I(0)$ e $F(0)$. 
Nesta modelagem foi considerada que todas as populações do modelo (2.1) são não negativas e a população imatura não excede a capacidade do meio. Desta forma, a região do espaço de estados deste sistema é definida por:

$$
\Gamma=\left\{(I, F) \in \mathbb{R}^{2} \mid F \geq 0 \text { e } 0 \leq I \leq C\right\} .
$$

A dependência em relação à precipitação dos parâmetros $\pi=\left(\phi, \alpha, \mu_{I}, \mu_{F}\right)$ é modelada por meio de leis de potências $[6,2]$ :

$$
\pi=\pi_{\min }+\left(\pi_{\max }-\pi_{\min }\right)\left(\frac{p-p_{\min }}{p_{\max }-p_{\min }}\right)^{r},
$$

em que $\pi$ representa genericamente os parâmetros entomológicos $\phi, \alpha, \mu_{I}, \mu_{F} ; \pi_{\min }$ e $\pi_{\max }$ representam, respectivamente, valores máximos e mínimos dos parâmetros entomológicos associados; $p_{\min }$ e $p_{\max }$ representam, respectivamente, valores máximos e mínimos da precipitação no horizonte de estudo; $p$ representa a precipitação acumulada em cada unidade de tempo e $r=0,7$.

As faixas dos parâmetros entomológicos com dependência da precipitação utilizados na simulação do modelo (2.1) estão descritos na Tabela 1. Os demais parâmetros foram considerados constantes ao longo de todo o horizonte de estudo $C=1[30] ; f=0,5[31] ; \gamma=0,5$ [11].

Tabela 1: Faixa em dias $^{-1}$ dos parâmetros entomológicos do modelo (2.1) com dependência da precipitação.

\begin{tabular}{ccccc}
\hline Parâmetro & Faixa & Parâmetro & Faixa & Fonte \\
\hline$\alpha$ & $0,02615-0,11612$ & $\mu_{I}$ & $0,01397-0,06001$ & {$[29]$} \\
$\phi$ & $1,060327-8,294997$ & $\mu_{F}$ & $0,035859-0,028773$ & {$[30]$} \\
\hline
\end{tabular}

O sistema (2.1) é não autônomo, pois parte de seus coeficientes dependem da precipitação que, por sua vez, depende do tempo. A precipitação será substituída por valores diários de uma determinada região geográfica e estas relações são exatas em cada período que as taxas permaneçam constantes. Desta forma, pode-se considerar o sistema autônomo a cada dia, sendo possível identificar dois pontos de equilíbrio, dados por:

1. Equilíbrio livre de infestação do mosquito:

$$
P_{0}=\left(I^{*}, F^{*}\right)=(0,0)
$$

2. Equilíbrio com infestação do mosquito:

$$
P_{1}=\left(I^{* *}, F^{* *}\right)=\left(C\left(1-\frac{\left(\alpha+\mu_{I}+u_{I}\right)\left(\mu_{F}+u_{F}\right)}{f \phi \gamma \alpha}\right), \frac{\gamma \alpha}{\left(\mu_{F}+u_{F}\right)} I^{* *}\right) .
$$

O método da matriz de próxima geração foi utilizado para determinar o número de descendentes basal $Q_{0}$ do modelo entomológico. Este método é similar ao desenvolvido em [26] para calcular 
o número de reprodutibilidade basal $R_{0}$ em modelos epidemiológicos. Neste caso, novos nascimentos do mosquito são tratados como novas infecções. O número de descendentes basal do mosquito representa o número médio de fêmeas que uma única fêmea gera ao longo de sua vida $[28,17]$. O sistema (2.1) pode ser reescrito como:

$$
\frac{d \mathbf{X}}{d t}=\frac{d}{d t}\left[\begin{array}{c}
I \\
F
\end{array}\right]=\left[\begin{array}{c}
f \phi\left(1-\frac{I}{C}\right) F \\
0
\end{array}\right]-\left[\begin{array}{c}
\left(\alpha+\mu_{I}+u_{I}\right) I \\
\left(\mu_{F}+u_{F}\right) F-\gamma \alpha I
\end{array}\right]=V-W
$$

em que $\mathbf{X}=(I, F)^{T}$ é o conjunto de todas as variáveis de estado do modelo. $V=V_{i}$ é o vetor de nascimento de ovos viáveis do mosquito em cada compartimento $i, i=1,2$. $W=W_{i}$ é o vetor de transição entre os compartimentos e pode ser decomposto como $W_{i}=W_{i}^{-}-W_{i}^{+}$em que $W_{i}^{+}$corresponde à taxa de transferência de indivíduos de outro compartimento para dentro do compartimento especificado e, analogamente, $W_{i}^{-}$corresponde à transferência para outro compartimento a partir do compartimento desejado [17].

As matrizes jacobianas de nascimento $\mathscr{V}$ e de transição $\mathscr{W}$ avaliadas no ponto equilíbrio livre de infestação do mosquito dado pela equação 2.3 são definidas por:

$$
\mathscr{V}=\left.\frac{\partial V}{\partial \mathbf{X}}\right|_{P_{0}}=\left[\begin{array}{cc}
0 & f \phi \\
0 & 0
\end{array}\right] \text { e } \mathscr{W}=\left.\frac{\partial W}{\partial \mathbf{X}}\right|_{P_{0}}=\left[\begin{array}{cc}
\left(\alpha+\mu_{I}+u_{I}\right) & 0 \\
-\gamma \alpha & \left(\mu_{F}+u_{F}\right)
\end{array}\right] .
$$

A matriz de próxima geração do sistema (2.5) é dada por:

$$
\mathscr{V} \mathscr{W}^{-1}=\left[\begin{array}{cc}
\frac{f \phi \gamma \alpha}{\left(\alpha+\mu_{I}+u_{I}\right)\left(\mu_{F}+u_{F}\right)} & \frac{f \phi}{\left(\mu_{F}+u_{F}\right)} \\
0 & 0
\end{array}\right]
$$

O raio espectral (maior autovalor em módulo) da matriz de próxima geração $\mathscr{V}^{-1}$, denotado por $\rho\left(V W^{-1}\right)$ é descrito por:

$$
\rho\left(V W^{-1}\right)=\frac{\gamma \alpha}{\left(\alpha+\mu_{I}+u_{I}\right)} \frac{f \phi}{\left(\mu_{F}+u_{F}\right)} .
$$

O número de descendentes basal do sistema (2.5) é dado por [26]:

$$
Q_{0}=\rho\left(V W^{-1}\right)=\frac{\gamma \alpha}{\left(\alpha+\mu_{I}+u_{I}\right)} \frac{f \phi}{\left(\mu_{F}+u_{F}\right)} .
$$

Note que o número de descendentes basal dado pela equação (2.9) não depende da capacidade do meio e $Q_{0} \geq 0$, pois todos os parâmetros do sistema são positivos. Biologicamente, $Q_{0}$ pode ser interpretado como o produto da fração da população imatura que sobrevive e emerge para a 
fase alada como fêmea $\left(\frac{\gamma \alpha}{\alpha+\mu_{I}+u_{I}}\right)$ pela fração de ovos viáveis gerados durante a sobrevida da fêmea $\left(\frac{f \phi}{\mu_{F}+u_{F}}\right)[10]$.

O ponto de equilíbrio com infestação do mosquito dado pela equação 2.4 pode ser reescrito em função de $Q_{0}$ :

$$
P_{1}=\left(C\left(1-\frac{1}{Q_{0}}\right), \frac{\gamma \alpha}{\left(\mu_{F}+u_{F}\right)} I^{*}\right)
$$

Observe que se $Q_{0}=1$, o ponto de equilíbrio com infestação do mosquito é biologicamente trivial, pois $P_{1}=P_{0}$. Se $Q_{0} \geq 1$, o ponto de equilíbrio com infestação do mosquito terá significado biológico.

O número de descendentes basal é importante no estudo da análise da estabilidade dos pontos de equilíbrio do sistema (2.5). Por meio dos Teoremas (1) e (2) foi possível verificar esta estabilidade. Este estudo foi realizado a partir do traço $T$ e do determinante $D$ da matriz jacobiana associada ao sistema, calculada nos valores correspondentes aos pontos de equilíbrio $P_{0}$ e $P_{1}$.

Theorem 1. O ponto de equilíbrio livre de infestação de mosquito, $P_{0}$ é uma sela instável se $Q_{0}>1$ e nó assintoticamente estável se $0<Q_{0}<1$.

Proof. A matriz jacobiana associada ao ponto de equilíbrio $P_{0}$ é descrita por:

$$
J_{P_{0}}=\left[\begin{array}{cc}
-\left(\alpha+\mu_{I}+u_{I}\right) & f \phi \\
\gamma \alpha & -\left(\mu_{F}+u_{F}\right)
\end{array}\right] .
$$

Os autovalores da matriz (2.11) são raízes do polinômio característico, representado por:

$$
p(\lambda)=\operatorname{det}\left(J_{P_{0}}-I \lambda\right)=\lambda^{2}+T_{J_{P_{0}}} \lambda+D_{J_{P_{0}}},
$$

em que $I$ é a matriz identidade de ordem 2, $T_{J_{P_{0}}}$ e $D_{J_{P_{0}}}$ são, respectivamente, o traço e o determinante da matriz (2.11), dados por:

$$
T_{J_{P_{0}}}=-\left[\left(\alpha+\mu_{I}+u_{I}\right)+\left(\mu_{F}+u_{F}\right)\right]<0 \text { e } D_{J_{P_{0}}}=\left(\alpha+\mu_{I}+u_{I}\right)\left(\mu_{F}+u_{F}\right)\left(1-Q_{0}\right) .
$$

Observe que se $Q_{0}>1$, o determinante de $J_{P_{0}}$ é negativo, o que implica que existem dois autovalores reais de $J_{P_{0}}$ com sinais opostos, e o ponto de equilíbrio $P_{0}$ é uma sela instável. Se $0<Q_{0}<1$, então o determinante de $J_{P_{0}}$ é positivo. Como o traço de $J_{P_{0}}$ é negativo e $T_{J_{P_{0}}}^{2}-4 D_{J_{P_{0}}}=\left[\left(\alpha+\mu_{I}+u_{I}\right)-\left(\mu_{F}+u_{F}\right)\right]^{2}+4 f \phi \gamma \alpha>0$, tem-se que os autovalores de $J_{P_{0}}$ são reais e negativos, e o ponto de equilíbrio $P_{0}$ é um nó assintoticamente estável.

Theorem 2. O ponto de equilíbrio com infestação de mosquito, $P_{1}$ é uma sela instável se $0<$ $Q_{0}<1$ e nó assintoticamente estável se $Q_{0}>1$. 
Proof. A matriz jacobiana associada ao ponto de equilíbrio $P_{1}$ pode ser escrita como

$$
J_{P_{1}}=\left[\begin{array}{cc}
\frac{-f \phi \gamma \alpha}{\left(\mu_{F}+u_{F}\right)} & \frac{f \phi}{Q_{0}} \\
\gamma \alpha & -\left(\mu_{F}+u_{F}\right)
\end{array}\right] .
$$

Os autovalores da matriz (2.13) são raízes do polinômio característico, representado por:

$$
p(\lambda)=\operatorname{det}\left(J_{P_{1}}-I \lambda\right)=\lambda^{2}+T_{J_{P_{1}}} \lambda+D_{J_{P_{1}}},
$$

em que $I$ é a matriz identidade de ordem 2, $T_{J_{P_{1}}}$ e $D_{J_{P_{1}}}$ são, respectivamente, o traço e o determinante da matriz (2.13), dados por:

$$
T_{J_{P_{1}}}=-\left[\frac{f \phi \gamma \alpha}{\left(\mu_{F}+u_{F}\right)}+\left(\mu_{F}+u_{F}\right)\right]<0 \text { e } D_{J_{P_{1}}}=f \phi \gamma \alpha\left(1-\frac{1}{Q_{0}}\right) .
$$

Observe que se $0<Q_{0}<1$, o determinante de $J_{P_{1}}$ é negativo, o que implica que existem dois autovalores reais de $J_{P_{1}}$ com sinais opostos, e o ponto de equilíbrio $P_{1}$ é uma sela instável. Se $Q_{0}>1$, então o determinante de $J_{P_{1}}$ é positivo. Como o traço de $J_{P_{1}}$ é negativo e $T\left(J_{P_{1}}\right)^{2}-4 D\left(J_{P_{1}}\right)=$ $\left[\frac{f \phi \gamma \alpha}{\left(\mu_{F}+u_{F}\right)}-\left(\mu_{F}+u_{F}\right)\right]^{2}+4\left(\alpha+\mu_{I}+u_{I}\right)\left(\mu_{F}+u_{F}\right)>0$, tem-se que os autovalores de $J_{P_{1}}$ são reais e negativos, e o ponto de equilíbrio $P_{1}$ é um nó assintoticamente estável.

O número de descendentes basal também auxilia ações de intervenção vetorial em uma determinada região geográfica. Se $Q_{0}>1$, as fêmeas do mosquito conseguem invadir e estabelecer sua população em uma determinada área geográfica, sendo esta área classifficada como infestada pelo vetor. Se $0<Q_{0}<1$, as fêmeas do mosquito não conseguem estabelecer sua população em uma determinada área geográfica e, ao longo do tempo, tenderá à extinção, ficando a localidade livre da infestação vetorial. Portanto, determinar a influência de cada parâmetro no valor de $Q_{0}$ é fundamental no impacto da configuração de infestação ou não infestação vetorial do sistema (2.5). Uma maneira de quantificar esta influência é por meio do índice de sensibilidade normalizado proposto por [4]. Este índice mostra o efeito local no número de descendentes basal do mosquito mediante a mudança de cada parâmetro genérico $\pi$ que compõem a equação (2.9). Neste cálculo o parâmetro genérico $\pi$ é considerado variável e os demais parâmetros constantes. O índice de sensibilidade normalizado, denotado por $\Phi_{\pi}^{Q_{0}}$, é definido por:

$$
\Phi_{\pi}^{Q_{0}}=\frac{\partial Q_{0}}{\partial \pi} \frac{\pi}{Q_{0}}
$$

em que $\pi$ representa genericamente um parâmetro de $Q_{0}$.

A expressão analítica da sensibilidade local de $Q_{0}$ em relação aos parâmetros $f, \phi, \gamma, \alpha, \mu_{I}, \mu_{F}$, $u_{I}$ e $u_{F}$ considerando os cenários sem e com intervenções de controle nas populações imatura e de fêmeas em fase de oviposição do A. aegypti estão ilustrados na Tabela 2.

Nos cenários sem e com intervenções de controle a taxa de oviposição, a taxa de desenvolvimento, a fração de imaturos que transformaram em fêmeas e a fração de ovos viáveis estão 
Tabela 2: Expressão analítica da sensibilidade normalizada do número de descendentes basal do Aedes aegypti sem e com intervenções de controle.

\begin{tabular}{ccc}
\hline Parâmetro & Sensibilidade Sem Controle & Sensibilidade Com Controle \\
\hline$f$ & +1 & +1 \\
\hline$\phi$ & $\frac{\mu_{I}}{\left(\alpha+\mu_{I}\right)} \in(0,1)$ & $\frac{\left(\mu_{I}+u_{I}\right)}{\left(\alpha+\mu_{I}+u_{I}\right)} \in(0,1)$ \\
\hline$\alpha$ & $-\frac{\mu_{I}}{\left(\alpha+\mu_{I}\right)} \in(-1,0)$ & $-\frac{\mu_{I}}{\left(\alpha+\mu_{I}+u_{I}\right)} \in(-1,0)$ \\
\hline$\mu_{I}$ & -1 & $-\frac{\mu_{F}}{\left(\mu_{F}+u_{F}\right)} \in(-1,0)$ \\
\hline$\mu_{F}$ & 0 & $-\frac{u_{I}}{\left(\alpha+\mu_{I}+u_{I}\right)} \in(-1,0)$ \\
\hline$u_{I}$ & & $-\frac{u_{F}}{\left(\mu_{F}+u_{F}\right)} \in(-1,0)$ \\
\hline$u_{F}$ & &
\end{tabular}

correlacionadas positivamente a $Q_{0}$. Enquanto que as taxas de mortalidade natural estão correlacionadas negativamente a $Q_{0}$. As taxas de mortalidade adicional estão correlacionadas negativamente a $Q_{0}$ durante as intervenções de controle. O efeito dos parâmetros $f, \phi$ e $\gamma$ são invariantes nos cenários sem e com intervenção de controle. No cenário sem intervenção de controle, os parâmetros que mais afetam $Q_{0}$ são a taxa de oviposição da fêmea, a fração de imaturos que transformaram em fêmeas, a fração de ovos viáveis e a taxa de mortalidade natural de fêmeas. Por exemplo, se $\mu_{F}$ é aumentado (ou diminuído) em $10 \%$, então $Q_{0}$ diminui (ou aumenta) também em $10 \%$. Impacto oposto sofre $Q_{0}$, variando-se $f$ e $\phi$. Com a implantação de intervenções de controle, ocorre um aumento da sensibilidade de $\alpha$ (correlacionada positivamente), $u_{I}$ (correlacionada negativamente) e $u_{F}$ (correlacionada negativamente) com $Q_{0}$ e uma redução da sensibilidade de $\mu_{I}$ (correlacionada negativamente) e $\mu_{F}$ (correlacionada negativamente) com $Q_{0}$. Portanto, intervenções de controle que reduzem a oviposição de ovos viáveis a se tornarem fêmeas adultas e que aumentem a mortalidade adicional dos mosquitos, poderão minimizar significativamente a infestação do A. aegypti em uma determinada área geográfica.

\section{CONTROLE}

Buscando investigar a taxa de mortalidade adicional e o horizonte mais adequado para realizar controle mecânico na população imatura e controle químico na população de fêmeas em fase de oviposição do A. aegypti foram consideradas três aplicações do respectivo controle nas intervenções Primavera-Verão, Verão e Verão-Outono em uma determinada área geográfica. Optou-se por não aplicar controle no último dia de cada estação. As intervenções foram realizadas das seguintes formas: (i) Na Primavera-Verão, uma aplicação ocorreu na Primavera e duas aplicações no Verão: a primeira aplicação foi realizada dentro dos últimos 45 dias da estação Primavera; a segunda aplicação foi realizada dentro dos primeiros 45 dias da estação Verão; e a 
terceira aplicação dentro dos últimos 45 dias da estação Verão; (ii) No Verão, as três aplicações de controle ocorreram no Verão: a primeira aplicação foi realizada dentro dos primeiros 30 dias da estação; a segunda aplicação foi realizada entre os dias 31 e 60 da estação; e a terceira aplicação foi realizada entre os dias 61 e 90 da estação; e (iii) No Verão-Outono, duas aplicações ocorreram no Verão e uma aplicação no Outono: a primeira aplicação foi realizada dentro dos primeiros 45 dias da estação Verão; a segunda aplicação foi realizada dentro dos últimos 45 dias da estação Verão; e a terceira aplicação foi realizada dentro dos primeiros 45 dias da estação Outono.

O tempo de aplicação do controle sobre as populações do mosquito foi definido como 7 dias [1]. Assim, durante 7 dias, foi aplicado um controle mecânico do tipo degrau com intensidade fixa $u_{I}^{i}$ na população imatura. O controle se inicia no tempo $t_{I}^{i}$ e dura até $\left(t_{I}^{i}+6\right)$ em cada uma das três aplicações da específica intervenção. Analogamente, foi aplicado um controle químico do tipo degrau com intensidade fixa $u_{F}^{i}$ na população de fêmeas em fase de oviposição. O controle se inicia no tempo $t_{F}^{i}$ e dura até $\left(t_{F}^{i}+6\right)$ em cada uma das três aplicações da específica intervenção.

A função objetivo $J$ do problema de otimização é dada pela equação (3.1) e apresenta a restrição (i) para a intervenção Primavera-Verão, (ii) para a intervenção Verão e (iii) para a intervenção Verão-Outono. Assume-se que a redução da população imatura desempenha um papel essencial no controle do A. aegypti. Assim, $J$ é formada pela combinação linear de integrais sobre o tempo de aplicação de controle de funções quadráticas, cujos coeficientes são os custos relativos de cada modalidade de controle. Portanto, $J$ é composta pela soma ponderada do custo com o controle (mecânico e pesticidas: parcelas 1 e 2) e do custo social de pessoas infectadas (medidas de acordo com o número de mosquitos imaturos e fêmeas fertilizadas em fase de oviposição: parcelas 3 e 4). Neste funcional as parcelas não representam custo financeiro. Cabe destacar que os termos quadráticos são usuais em problemas de otimização dinâmica, atuando como uma ampliação de efeitos de grande variação das variáveis [25].

$$
\begin{gathered}
\min _{u_{I}^{i}, t_{I}^{i}, u_{F}^{i}, t_{F}^{i}} J\left(u_{I}^{i}, t_{I}^{i}, u_{F}^{i}, t_{F}^{i}\right)=\frac{1}{2}\left(c_{1} \int_{t_{I}^{i}}^{t_{F}^{i}}\left(u_{I}^{i}\right)^{2} d t+c_{2} \int_{t_{I}^{i}}^{t_{F}^{i}}\left(u_{F}^{i}\right)^{2} d t+\right. \\
\left.c_{3} \int_{0}^{364} I^{2} d t+c_{4} \int_{0}^{364} F^{2} d t\right), i=1,2,3 ;
\end{gathered}
$$

(i) Primavera - Verão.

$$
\text { s.a: }\left\{\begin{array} { l } 
{ \text { Modelo } ( 2 . 1 ) ; } \\
{ 0 \leqslant u _ { I } ^ { i } , u _ { F } ^ { i } \leqslant 1 ; } \\
{ 4 6 \leqslant t _ { I } ^ { 1 } , t _ { F } ^ { 1 } \leqslant 8 4 ; } \\
{ 9 2 \leqslant t _ { I } ^ { 2 } , t _ { F } ^ { 2 } \leqslant 1 3 0 ; } \\
{ 1 3 7 \leqslant t _ { I } ^ { 3 } , t _ { F } ^ { 3 } \leqslant 1 7 5 . }
\end{array} \quad \text { s.a: } \left\{\begin{array} { l } 
{ \text { Modelo } ( 2 . 1 ) ; } \\
{ 0 \leqslant u _ { I } ^ { i } , u _ { F } ^ { i } \leqslant 1 ; } \\
{ 1 \leqslant t _ { I } ^ { 1 } , t _ { F } ^ { 1 } \leqslant 2 4 ; } \\
{ 3 1 \leqslant t _ { I } ^ { 2 } , t _ { F } ^ { 2 } \leqslant 5 4 } \\
{ 6 1 \leqslant t _ { I } ^ { 3 } , t _ { F } ^ { 3 } \leqslant 8 4 . }
\end{array} \quad \text { s.a: } \left\{\begin{array}{l}
\text { Modelo }(2.1) ; \\
0 \leqslant u_{I}^{i}, u_{F}^{i} \leqslant 1 ; \\
1 \leqslant t_{I}^{1}, t_{F}^{1} \leqslant 39 ; \\
46 \leqslant t_{I}^{2}, t_{F}^{2} \leqslant 84 ; \\
92 \leqslant t_{I}^{3}, t_{F}^{3} \leqslant 130 .
\end{array}\right.\right.\right.
$$

sendo que $c_{1}$ e $c_{2}$ representam o custo relativo à intensidade de controle adicional aplicado na população imatura e na população de fêmeas em fase de oviposição, respectivamente; $c_{3}$ e $c_{4}$ representam o custo social associado a população imatura e a população de fêmeas em fase de oviposição, respectivamente. Neste trabalho adotou-se $c_{i}=1$, em que $i=1,2,3,4$.

Tend. Mat. Apl. Comput., 20, N. 1 (2019) 
Observe que a função objetivo do problema de otimização apresenta descontinuidade nas variáveis, cuja restrição é um sistema dinâmico não-linear dependente da precipitação de uma determinada área geográfica. Este fato torna difícil a solução analítica ou por métodos determinísticos, o que justifica o uso de heurísticas. Os algoritmos baseados em inteligência computacional não garantem a otimalidade global da solução, mas as soluções geralmente são de boa qualidade. Dentre estes algoritmos destaca-se os Algoritmos Genéticos, que são caracterizados pela evolução de uma população de possíveis soluções que obedecem regras estocásticas de seleção, cruzamento e mutação ao longo de suas gerações [16], [15], [14].

No presente trabalho, a busca de soluções do problema de otimização foi obtida por meio do Algoritmo Genético Real Polarizado - AGRP [24, 23]. O AGRP é de fácil implementação e o seu funcionamento é o seguinte:

- Cada parâmetro de otimização é descrito por uma variável real, sendo o conjunto de parâmetros armazenado em um vetor (indivíduo) com 12 posições $\left[u_{I}^{1}, t_{I}^{1}, u_{I}^{2}, t_{I}^{2}, u_{I}^{3}, t_{I}^{3}, u_{F}^{1}, t_{F}^{1}, u_{F}^{2}, t_{F}^{2}, u_{F}^{3}, t_{F}^{3}\right]$.

- A faixa admissível para cada coordenada do vetor de parâmetros deve ser respeitada.

- A inicialização do algoritmo se deu pela geração aleatória de uma população com $N=200$ vetores (indivíduos) com 12 posições dentro das faixas admissíveis.

- A cada geração do AGRP uma nova população com mesmo número de indivíduos foi gerada após a sequência de operações:

- Cruzamento Real-Polarizado: A cada geração divide-se a população corrente em duas metades. Para cada par formado, por exemplo $x_{1}$ e $x_{2}$ indivíduos (pais) foi verificado se ocorre cruzamento ou não, com probabilidade de 0,90 . Em caso afirmativo, são gerados dois novos indivíduos (filhos), dados por $x=\alpha x_{1}+(1-\alpha) x_{2},-0,1<\alpha<1,1$ e $J\left(x_{2}\right)<J\left(x_{1}\right)$. Para a geração de $\alpha$ é verificado se o cruzamento será polarizado ou não-polarizado, com probabilidade de 0,30 para ser polarizado. Em caso afirmativo, para um dos novos indivíduos (filhos) escolhe-se $\alpha=1,4 \beta_{1} \beta_{2}-0,2$, sendo $\beta_{1}$ e $\beta_{2}$ escolhidos com maior chance do filho estar mais perto do melhor pai, com distribuição de probabilidade uniforme no intervalo [0 1]. O outro indivíduo (filho) será sempre escolhido sem polarização. Cada novo indivíduo (filho) estará sobre um mesmo segmento de reta com extremos localizados de forma a ultrapassar em 0,2 os indivíduos (pais). Desta forma, torna-se possível que os novos indivíduos (filhos) estejam fora das faixas admissíveis de parâmetros. Caso isso ocorra, será realizada uma operação de reflexão do indivíduo (filho) $x$ para o interior da região admissível. A reflexão no limite inferior é dada por $x_{r}{ }^{I}=x_{L}+\left|x-x_{L}\right|$ e no limite superior é dada por $x_{r}{ }^{S}=x_{U}+\left|x_{U}-x\right|$. Caso a operação de cruzamento não seja polarizada, adota-se $\alpha$ com distribuição uniforme de probabilidade dentro do intervalo de valores possíveis para ambos os novos indivíduos (filhos) gerados.

- Mutação Gaussiana: Em cada geração determina-se para cada indivíduo da população se ele sofrerá ou não mutação, com probabilidade 0,05 . Em caso afirmativo, será somado ao indivíduo $x$ um vetor $\delta$ cujas componentes são dadas por $\delta_{i}=0,05 \beta_{i}\left(x_{R}\right)_{i}$, sendo $\beta_{i}$ um número aleatório com distribuição gaussiana, média zero e variância um, e $x_{R}$ corresponde ao vetor de diferença entre os valores máximos e mínimos dos parâmetros. 
- Avaliação: Cada indivíduo da população corrente é avaliado na função objetivo $J$ do problema de otimização que considera, para o cálculo das variáveis, a evolução numérica do sistema de equações diferenciais.

- Função de Ajuste: Em cada geração calcula-se para cada indivíduo da população um valor de função de ajuste igual à proposta por [14]. Foi adotado o valor de 1.8 para o fator de dispersão na função de ajuste.

- Seleção por Roleta: Em cada geração realiza-se uma seleção de 200 indivíduos dentre os 200 indivíduos existentes da população, sendo que cada indivíduo pode ser selecionado mais de uma vez. A probabilidade de um indivíduo ser selecionado corresponde ao valor da fração de sua função de ajuste em relação à soma das funções de ajuste dos demais indivíduos da população.

- Elitismo: Se o melhor indivíduo da população corrente não tenha sido selecionado para compor a nova população, ele será introduzido nela, com a exclusão de um indivíduo qualquer, escolhido aleatoriamente.

- O término do algoritmo ocorre quando extrapola-se o número máximo permitido de gerações. Foi adotado o valor de 600 para o número máximo de gerações.

A eficiência das três formas de intervenções de controle realizadas nas populações imatura e de fêmeas em fase de oviposição do mosquito, denotada por $\Sigma$, foi calculada de acordo com [32] e é descrita por:

$$
\Sigma=\left(1-\left(\frac{A_{c}}{A_{s}}\right)\right) \times 100
$$

em que $A_{c}$ é a área abaixo da curva da população do mosquito com intervenção de controle e $A_{s}$ a área abaixo da curva da população do mosquito sem intervenção de controle.

\section{SIMULAÇÃo}

Para avaliar a dinâmica do sistema (2.1) com e sem intervenção de controle utilizou-se dados amostrais de precipitação acumulada $(\mathrm{mm})$ por dia da cidade de Lavras (Minas Gerais, Brasil) do Instituto Nacional de Pesquisas Espaciais. Adotou-se a média diária de 15 anos de precipitação desta cidade, correspondendo aos anos de 2002 a 2016. Cada estação do ano contém 91 dias, totalizando um ano com 364 dias. Para esta configuração o mês de fevereiro contém 28 dias e foram excluídos os dados de precipitação do dia 31 de dezembro. A simulação da dinâmica das populações do sistema (2.1) foi obtida por meio do algoritmo de Runge-Kutta de quarta ordem, com passo $h=0,01$. Este método é amplamente utilizado dentro da classe de métodos numéricos para resolver uma equação diferencial e encontra-se detalhado em [12]. A condição inicial deste sistema foi adotada como o valor do ponto de equilíbrio com infestação do mosquito referente ao primeiro dia do horizonte de estudo.

Na validação do modelo (2.1) confrontou-se os dados simulados de fêmeas em fase de oviposição com dados amostrais de Índice Médio de Fêmeas Aedes - IMFA no período referente as semanas epidemiológicas (9 - 52) de 2009 a (1 - 52) de 2010 da cidade de Lavras. O IMFA corresponde à razão entre o número de fêmeas de Aedes grávidas capturadas semanalmente pela armadilha MosquiTRAP ${ }^{\circledR}$ e o número total de armadilhas instaladas na região de estudo [9]. Os dados amostrais de IMFA foram coletados semanalmente pelo sistema de Monitoramento Inteligente do Aedes (MI-Aedes) por meio de 245 armadilhas MosquiTRAPs ${ }^{\circledR}$ espalhadas na área urbana de Lavras, sendo que cada armadilha MosquiTRAP ${ }^{\circledR}$ foi instalada no peridomicílio 

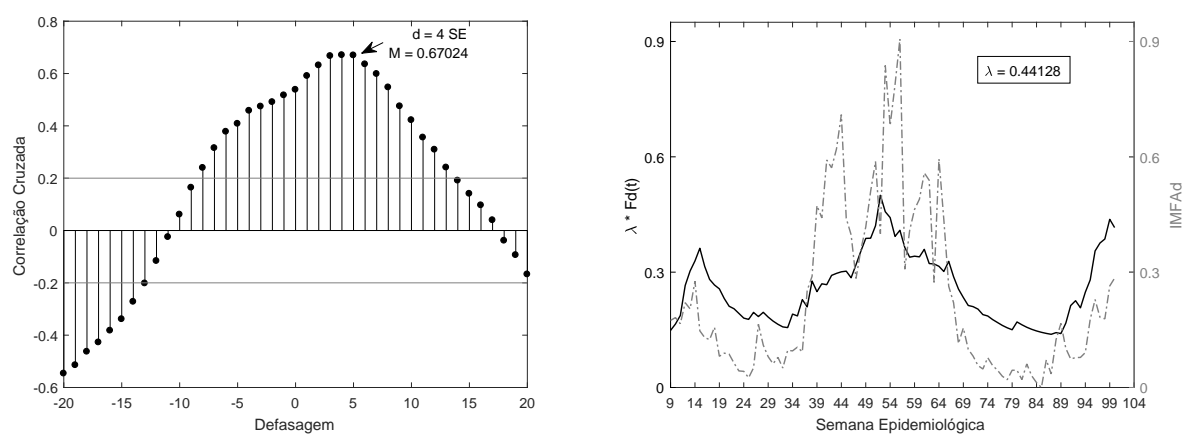

Figura 1: Correlação cruzada e comparação entre os dados simulados de fêmeas em fase de oviposição do modelo (2.1) e os dados amostrais de Índice Médio de Fêmeas Aedes com deslocamento calculado a partir correlação cruzada e fator escala no horizonte de estudo da cidade de Lavras (Minas Gerais, Brasil).

de uma residência, respeitando a distância mínima padrão de 250 metros entre as armadilhas e vistoriadas semanalmente. O erro quadrático médio $s^{2}$ existente entre os dados simulados de fêmeas em fase de oviposição e dados amostrais de IMFA referente ao horizonte de estudo foi descrito por:

$$
s^{2}=\frac{1}{2 N} \sum_{t=1}^{N}(I(t)-\lambda F(t-d))^{2} \text { e } \lambda=\frac{\sum_{t=1}^{N}(F(t-d) I(t))}{\sum_{t=1}^{N}(F(t-d))^{2}}
$$

em que $N$ é o número total de semanas epidemiológicas do horizonte de estudo; $I(t)$ corresponde aos dados amostrais de IMFA; $F(t)$ corresponde aos dados simulados de fêmeas em fase de oviposição; $d$ é a defasagem positiva correspondente à máxima correlação cruzada entre os dados; e $\lambda$ é um fator positivo que ajusta verticalmente a escala relativa dos dados simulados de fêmeas em fase de oviposição com dados amostrais de IMFA após a correlação cruzada.

A Figura (1) ilustra a comparação qualitativa entre os dados simulados de fêmeas em fase de oviposição do modelo (2.1) e os dados amostrais de IMFA, com deslocamento calculado a partir correlação cruzada e fator escala para a cidade de Lavras. A evolução da população de fêmeas seguiu a tendência de evolução do IMFA com um atraso médio entre os picos e os vales de 4 semanas epidemiológicas e $s^{2}=0,01305$. Segundo a classificação de [8], a intensidade desta correlação foi moderada $(M=0,67024)$. Esta comparação pode ser considerada satisfatória, uma vez que foi considerado a influência apenas da precipitação na dinâmica das populações do A. aegypti descritas no modelo (2.1) e os dados amostrais de IMFA e de precipitação podem sofrer influência de diversas formas, como humanas e mecânicas.

O AGRP foi implementado na linguagem de programação C e executado no Cluster do Laboratório de Computação de Alto Desempenho do Centro Federal de Educação Tecnológica de Minas Gerais. Neste experimento foram realizadas 30 execuções do algoritmo para cada uma das três formas de intervenções de controle, sendo que cada execução demorou em média 60 segundos. Os parâmetros utilizados durante a execução do AGRP foram escolhidos empiricamente após a realização de inúmeros testes computacionais. As curvas da Figura 2 justificam que esta escolha leva uma convergência do valor do funcional ótimo dado pela equação (3.1) em todas as três formas de intervenções de controle do mosquito. 
(a) Primavera - Verão.

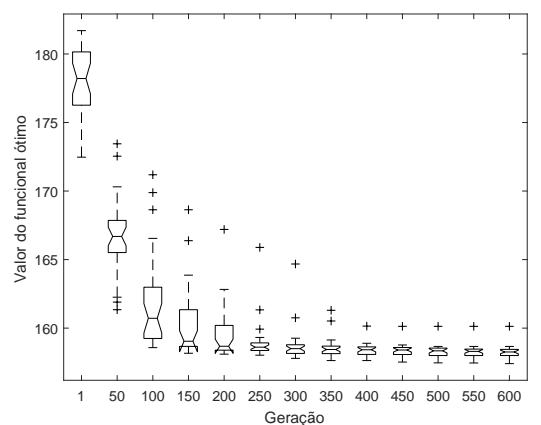

(b) Verão.

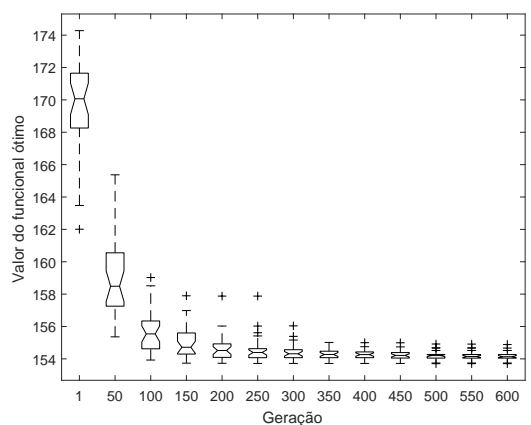

(c) Verão - Outono.

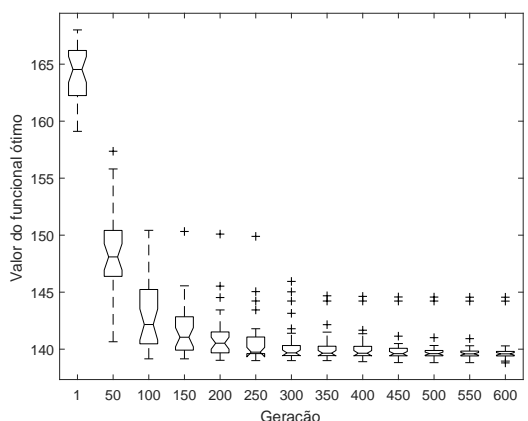

Figura 2: Evolução do valor do funcional ótimo ao longo das gerações após as 30 execuções do Algoritmo Genético Real Polarizado considerando cada uma das formas de intervenções de controle do Aedes aegypti no horizonte de estudo da cidade de Lavras (Minas Gerais, Brasil).
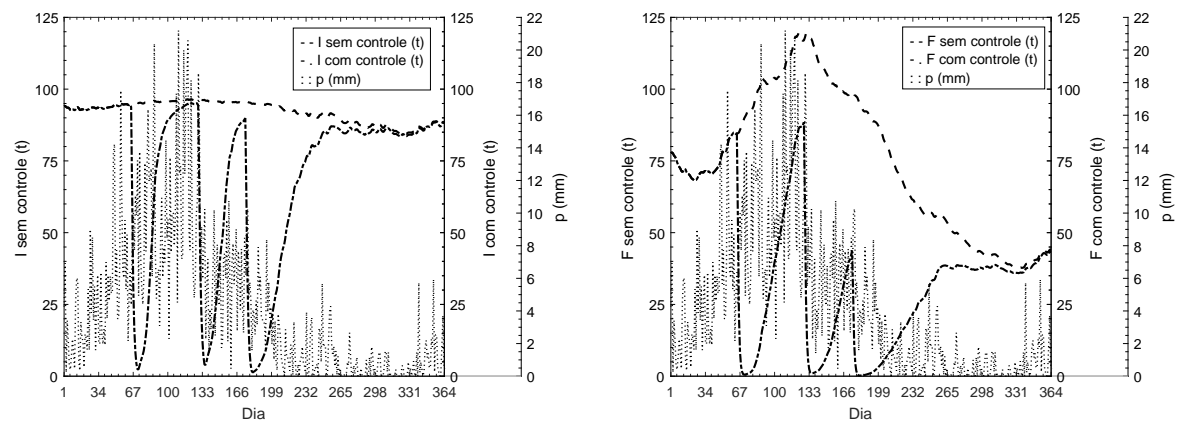

Figura 3: Evolução das populações imatura e de fêmeas em fase de oviposição do Aedes aegypti no cenário sem e com controle durante a intervenção PrimaveraVerão no horizonte de estudo da cidade de Lavras (Minas Gerais, Brasil). 

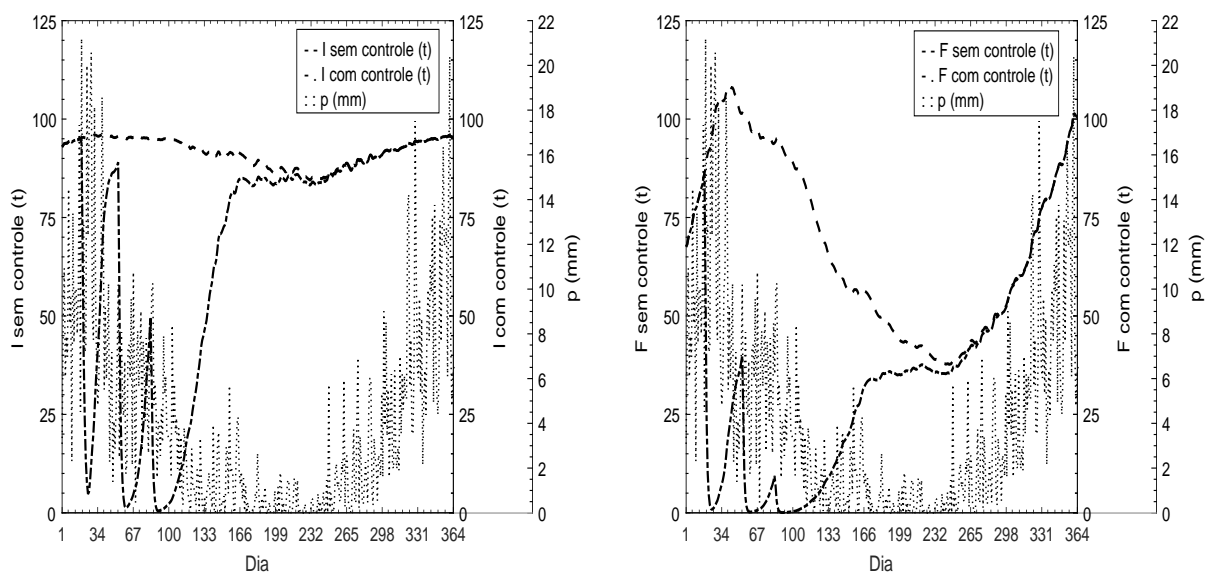

Figura 4: Evolução das populações imatura e de fêmeas em fase de oviposição do Aedes aegypti no cenário sem e com controle durante a intervenção Verão no horizonte de estudo da cidade de Lavras (Minas Gerais, Brasil).
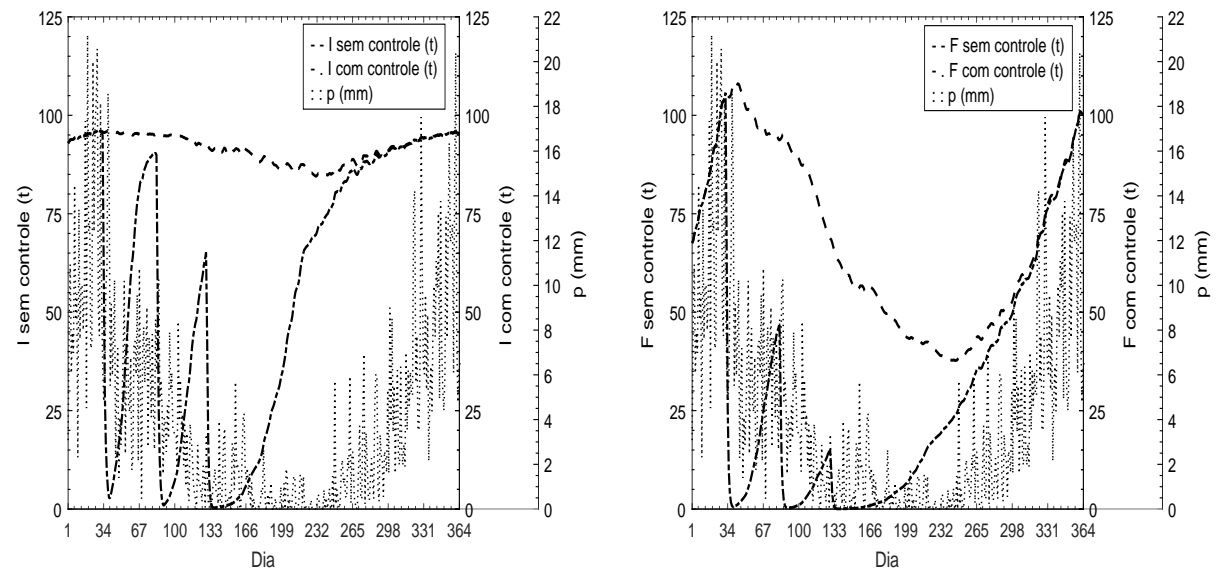

Figura 5: Evolução das populações da fase imatura e de fêmeas em fase de oviposição do Aedes aegypti no cenário sem e com controle durante a intervenção Verão-Outono no horizonte de estudo da cidade de Lavras (Minas Gerais, Brasil). 
Tabela 3: Valor do funcional ótimo e da eficiência das três aplicações de controle nas populações imatura e de fêmeas em fase de oviposição do Aedes aegypti durante as intervenções Primavera-Verão, Verão e Verão-Outono no horizonte de estudo da cidade de Lavras (Minas Gerais, Brasil).

\begin{tabular}{cccc}
\hline Intervenção Total & $J^{*}$ & $\Sigma_{I}(\%)$ & $\Sigma_{F}(\%)$ \\
\hline Primavera-Verão & 157,41 & 22,99 & 51,19 \\
Verão & 153,71 & 25,99 & 47,73 \\
Verão-Outono & 138,80 & 36,38 & 53,11 \\
\hline
\end{tabular}

O melhor valor obtido das soluções do problema de otimização mono-objetivo foi adotado como o menor valor de função objetivo encontrado dentre as 30 execuções do AGRP em cada uma das intervenções de controle. A Tabela 4 ilustra o valor ótimo obtido para as variáveis de decisão em cada uma das três aplicações de controle nas populações imatura e de fêmeas em fase de oviposição do A. aegypti durante as intervenções Primavera-Verão, Verão e Verão-Outono. O valor do funcional ótimo $\left(J^{*}\right)$ e da eficiência $(\Sigma)$ das três intervenções de controle estão representados na Tabela 3.

Tabela 4: Valor ótimo do problema de otimização em cada aplicação de controle nas populações imatura e de fêmeas em fase de oviposição do Aedes aegypti durante as intervenções Primavera-Verão, Verão e Verão-Outono no horizonte de estudo da cidade de Lavras (Minas Gerais, Brasil).

\begin{tabular}{ccccc}
\hline Intervenção 1 & $u_{I}^{1}$ & $t_{I}^{1}$ & $u_{F}^{1}$ & $t_{F}^{1}$ \\
\hline Primavera-Verão & 0,97 & 66 & 0,99 & 64 \\
Verão & 0.80 & 20 & 0,96 & 19 \\
Verão-Outono & 0,99 & 34 & 0,99 & 33 \\
\hline Intervenção 2 & $u_{I}^{2}$ & $t_{I}^{2}$ & $u_{F}^{2}$ & $t_{F}^{2}$ \\
\hline Primavera-Verão & 0,87 & 129 & 0,88 & 129 \\
Verão & 0,99 & 54 & 0,99 & 53 \\
Verão-Outono & 0,99 & 84 & 0,99 & 83 \\
\hline Intervenção 3 & $u_{I}^{3}$ & $t_{I}^{3}$ & $u_{F}^{3}$ & $t_{F}^{3}$ \\
\hline Primavera-Verão & 0,99 & 175 & 0,99 & 175 \\
Verão & 0,99 & 84 & 0,99 & 84 \\
Verão-Outono & 0,99 & 130 & 0,99 & 130 \\
\hline
\end{tabular}

É possível observar que o menor valor de funcional ótimo e a melhor eficiência do controle tanto na população imatura quanto na população de fêmeas em fase de oviposição do A. aegypti ocorreram na intervenção Verão-Outono. Neste período do ano, a quantidade de controle aplicada em ambas as populações do mosquito foram intensas. A intervenção Verão apresentou um menor valor de funcional ótimo e eficiência na população imatura do mosquito quando comparado com a intervenção Primavera-Verão. 
(a) Primavera - Verão.

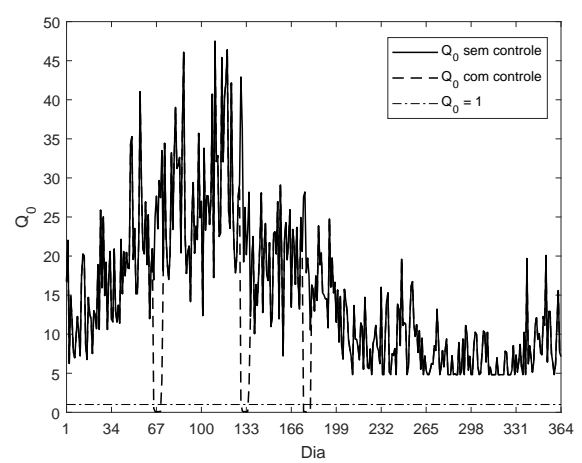

(b) Verão.

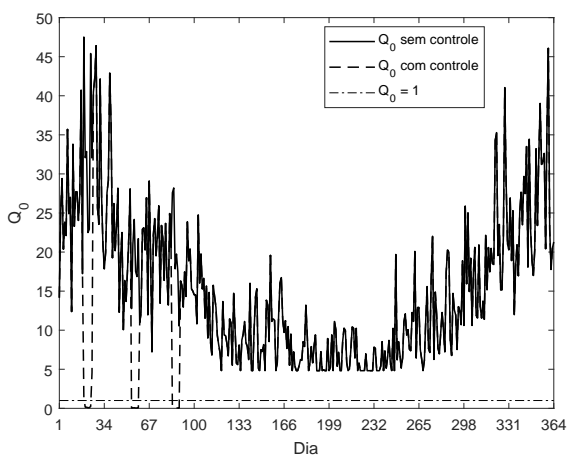

(c) Verão - Outono.

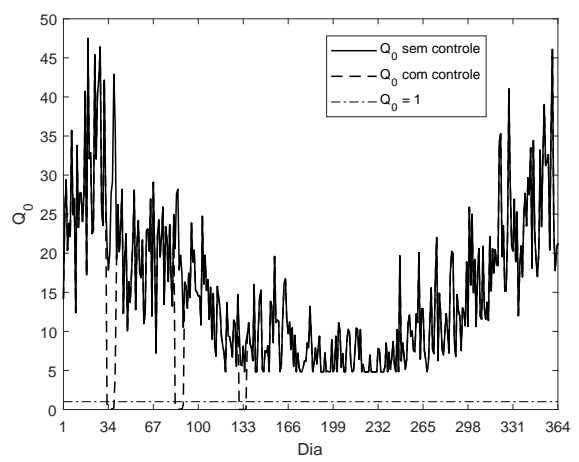

Figura 6: Evolução do número de descendentes basal do Aedes aegypti no cenário sem e com controle durante as intervenções Primavera-Verão, Verão e Verão-Outono no horizonte de estudo da cidade de Lavras (Minas Gerais, Brasil).

As curvas das Figuras (3) - (5) ilustram a comparação da evolução das populações do modelo (2.1) no cenário sem e com as três aplicações de controle nas intervenções Primavera-Verão, Verão, Verão-Outono no horizonte de estudo da cidade de Lavras (Minas Gerais, Brasil). No cenário sem intervenção de controle percebe-se que uma pequena variação na precipitação é o suficiente para manter a população imatura elevada e a população de fêmeas em fase de oviposição segue a tendência de evolução da precipitação. Nas intervenções com controle, foi utilizado o respectivo valor ótimo das variáveis de decisão gerados no AGRP, no qual verificou-se uma redução significativa das populações imatura e de fêmeas em fase de oviposição do mosquito. O efeito da última aplicação de controle em cada intervenção foi mais prolongado ao longo do horizonte posterior ao seu término de aplicação. Nas aplicações Verão e Verão-Outono o pico da população de fêmeas em fase de oviposição foi reduzido em mais de $90 \%$, enquanto que na intervenção Primavera-Verão esta redução foi de aproximadamente $30 \%$.

As curvas da Figura 6 ilustram a comparação da evolução do número de descendentes basal do A. aegypti no cenário sem e com as três aplicações de controle nas intervenções Primavera-Verão, Verão, Verão-Outono no horizonte de estudo da cidade de Lavras (Minas Gerais, Brasil). No cenário sem controle, o mosquito encontrou condições favoráveis à sua infestação $\left(Q_{0}>1\right)$ em todo horizonte. Durante as intervenções de 
controle, observou-se que número de descendentes basal atingiu um valor menor que um $\left(Q_{0}<1\right)$, ou seja, resultou horizontes livres de infestação de A. aegypti.

\title{
5 CONCLUSÕES
}

No cenário sem intervenção de controle, a evolução do número de descendentes basal do mosquito seguiu a tendência da precipitação e o vetor conseguiu infestar as cidades ao longo do horizonte de estudo. Este fato pode estar relacionado à disponibilidade contínua de criadouros artificiais viáveis ao mosquito nesta cidade. O número elevado de $A$. aegypti em uma determinada área potencializa a dispersão de suas arboviroses e, consequentemente, produz prejuízo social e econômico à sociedade. A utilização de medidas de controle no ciclo de vida do mosquito é uma forma de reduzir o nível de infestação do vetor em uma determinada localidade. Diante deste fato, o resultado obtido nas simulações do problema de otimização sugere que as ações de controle no ciclo de vida do mosquito nas intervenções Primavera-Verão, Verão e Verão-Outono em intervalos regulares de tempo agiram como uma medida preventiva contra a infestação do mosquito, sendo que a intervenção de controle realizada no Verão-Outono apresentou o melhor custo-benefício.

\section{ACKNOWLEDGMENTS}

Trabalho financiado pela CAPES e apresentado no XXXVII Congresso Nacional de Matemática Aplicada e Computacional.

\begin{abstract}
Arboviruses transmitted by Aedes aegypti such as dengue, chikungunya, zika and yellow fever have affected a growing part of the world population over the years. Since there are no vaccines for all arboviruses transmitted by this vector, population vector control is the approach considered feasible to reduce the spread of arboviruses. The present work has the goal of studying and comparing the efficacy of three mechanical control applications in the immature population (eggs, larvae and pupae) and to perform three chemical control applications on the population of females in the oviposition phase of A. aegypti. The control is applied during 7 days in different times of the year: Spring-Summer, Summer and Summer-Autumn. The entomological model that describes the dynamics of the phases of A. aegypti has precipitation-dependent parameters. The population of females in the oviposition phase of the model was confronted with sample data of Mean Female Aedes Index collected through 245 traps MosquiTRAP ${ }^{\circledR}$ scattered in the urban area of the city of Lavras. The Real-Biased Genetic Algorithm was used in order to optimize the intensity and the initial time of application of the step control in relation to control costs and costs related to mosquito infestation. During the application of the three forms of control interventions, the number of mosquitoes decreased over time and the summer-autumn intervention presented the best efficiency and the lowest cost. In addition, the number of mosquito basal offspring had a negative impact on the parameters related to mortality and an opposite impact of the other entomological parameters of the mosquito.
\end{abstract}

Keywords: control, Aedes aegypti, Real-Biased Genetic Algorithm. 


\section{REFERÊNCIAS}

[1] L.S. Barsante. "Dependência entre pluviosidade e população de fêmeas Aedes aegypti grávidas descritas através de um sistema dinâmico não linear". mathesis, Centro Federal de Educação Tecnológica de Minas Gerais, Belo Horizonte, MG (2012).

[2] L.S. Barsante, R.T.N. Cardoso, J.L. Acebal, C.A. Silva \& Á.E. Eiras. Modelo Entomológico Determinístico sob Efeito da Pluviosidade para o Aedes aegypti e o Aedes albopictus. Proceeding Series of the Brazilian Society of Computational and Applied Mathematics, 5(1) (2017). doi:https: //doi.org/10.5540/03.2017.005.01.0066.

[3] L.S. Barsante, R.T.N. Cardoso, J.L. Acebal, C.A. Silva \& Á.E. Eiras. Modelo Entomológico Determinístico sob Efeito da Pluviosidade para o Aedes aegypti e o Aedes albopictus. Tendência em Matemática Aplicada e Computacional, 19(2) (2018), 289-303. doi:10.5540/tema.2018.019.02.0289.

[4] J. Cariboni, D. Gatelli, R. Liska \& A. Saltelli. The role of sensitivity analysis in ecological modelling. Ecological Modelling, 203 (2007), 167-182.

[5] R.C. Catão. "Dengue no Brasil: abordagem geográfica na escala nacional”. São Paulo - SP (2012).

[6] F.S. Cordeiro. "Modelo preditivo para a população de mosquitos Aedes aegypti sob influência de variáveis climáticas: temperatura e pluviosidade”. mathesis, Centro Federal de Educação Tecnológica de Minas Gerais, Belo Horizonte, MG (2015).

[7] V.A. Costa \& J.P.G. Silva. Associação entre os casos notificados e variáveis climáticas da dengue no município de Pirapora (MG) entre os anos de 2010 a 2011. Caminhos de Geografia, 14(45) (2013), $161-171$.

[8] C.P. Dancey \& J. Reidy. "Estatística sem matemática para psicologia". Porto alegre: Artes médicas ed. (2006).

[9] Á.E. Eiras \& M.C. Resende. Preliminary evaluation of the Dengue-MI technology for Aedes aegypti monitoring and control. Cad. Saúde Pública, 25(1) (2009), S45 - S58.

[10] L. Esteva, G. Rivas \& H.M. Yang. Modelling parasitism and predation of mosquitoes by water mites. Journal of Mathematical Biology, 53 (2006), 540-555. doi:10.1007/s00285-006-0020-3.

[11] L. Esteva \& H.M. Yang. Mathematical model to assess the control of Aedes aegypti mosquitoes by the sterile insect technique. Mathematical Biosciences, 198 (2005), 132-147.

[12] F.F.C. Filho. "Algoritmo Numéricos". LTC, Rio de Janeiro, Brasil, 2 ed. (2007).

[13] H.O. Florentino, D.R. Cantane, C.A. Reis, D. Colón \& S.S.R.F. Rosa. Algoritmo de Busca em Vizinhança Variável para Determinação de Controle Otimizado para a População de Aedes aegypti. Proceeding Series of the Brazilian Society of Applied and Computational Mathematics, 6(1) (2018). doi:10.5540/03.2018.006.01.0308.

[14] D.E. Goldberg. "Genetic algorithms in search, optimization, and machine learning" (1989).

[15] D.E. Goldberg \& J.H. Holland. Genetic Algorithms and Machine Learning. Machine Learning, 3 (1988), 95-99. 
[16] J.H. Holland. "Adaptation in natural and artificial systems" (1975).

[17] A.M. Lutambi, M.A. Penny, T. Smith \& N. Chitnis. Mathematical modelling of mosquito dispersal in a heterogeneous environment. Mathematical Biosciences, 241 (2013), 198-216.

[18] N. Mladenović \& P. Hansen. Variable neighborhood search. Computers \& Operations Research, 24(11) (1997), 1097-1100.

[19] J.F.A. Montoya, A.M. Loaiza \& C.A.A. Muñoz. Population dynamics of Aedes aegypti considering quiescence. Applied Mathematical Sciences, 9(74) (2015), 3659-3668.

[20] J. Patterson, M. Sammon \& M. Garg. Dengue, Zika and Chikungunya: Emerging Arboviruses in the New World. Western Journal of Emergency Medicine, 17(6) (2016), 671-679. doi:0.5811/westjem. 2016.9.30904.

[21] L.E.M. Santos, C.A. Silva, L.S. Barsante \& J.L.A. Fernandes. Inteligência computacional aplicada ao controle do Aedes Aegypti. Revista Interdisciplinar de Pesquisa em Engenharia - RIPE, 2(9) (2016).

[22] H.H.G. Silva \& I.G. Silva. Influência do período de quiescência dos ovos sobre o ciclo de vida de $A e$ des aegypti (Linnaeus, 1762) (Diptera: Culicidae) em condições de laboratório. Revista da Sociedade Brasileira de Medicina Tropical, 32(4) (1999), 349-355.

[23] R.H.C. Takahashi. "Otimização Escalar", volume 2. Universidade Federal de Minas Gerais, Belo Horizonte, Minas Gerais, Brasil (2007).

[24] R.H.C. Takahashi, J.A. Vasconcelos, J.A. Ramírez \& L. Krahenbuhl. A Multiobjective Methodology for Evaluating Genetic Operators. IEEE Transactions on Magnetics, 39(3) (2003), 1321-1324.

[25] R.C.A. Thomé, H.M. Yang \& L. Esteva. Optimal control of Aedes aegypti mosquitoes by the sterile insect technique and insecticide. Mathematical Biosciences, 223 (2010), 12-23.

[26] P. van den Driessche \& J. Watmough. Reproduction numbers and sub-threshold endemic equilibria for compartmental models of disease transmission. Mathematical Biosciences, 180(1) (2002), 29-48.

[27] F.S. Veríssimo, L.S. Barsante, J.L. Acebal \& R.T.N. Cardoso. Modelagem e controle do Aedes aegypti durante as estações do ano através do Algoritmo Genético. Proceeding Series of the Brazilian Society of Applied and Computational Mathematics, 4(1) (2016). doi:10.5540/03.2016.004.01.0062.

[28] M.T. White, J.T. Griffin, T.S. Churcher, N.M. Ferguson, M.G. Basanez \& A. Ghani. Modelling the impact of vector control interventions on Anopheles gambiae population dynamics. Parasites \& Vectors, 4(153) (2011).

[29] H.M. Yang. Assessing the Influence of Quiescence Eggs on the Dynamics of Mosquito Aedes aegypti. Applied Mathematics, 5 (2014), 2696-2711.

[30] H.M. Yang. The transovarial transmission in the dynamics of dengue infection: Epidemiological implications and thresholds. Mathematical Biosciences, 286 (2017), 1-15.

[31] H.M. Yang, J.L. Boldrini, A.C. Fassoni, L.F.S. Freitas, M.C. Gomez, K.K.B. Lima, V.R. Andrade \& A.R.R. Freitas. Fitting the incidence data from the city of Campinas, Brazil, based on dengue transmission modellings considering time-dependent entomological parameters. Plos One, 11(3) (2016), e0152186. 
[32] H.M. Yang \& C.P. Ferreira. Assessing the effects of vector control on dengue transmission. Applied Mathematics and Computation, 198 (2008), 401-413.

[33] X.S. Yang. "Nature-Inspired Metaheuristic Algorithm". 1 ed. (2008), 116 pp. 